\title{
Upregulation of adenylate cyclase 3 (ADCY3) increases the tumorigenic potential of cells by activating the CREB pathway
}

\author{
Seung-Hyun Hong ${ }^{1, *}$, Sung-Ho Goh ${ }^{2, *}$, Sang Jin Lee ${ }^{3}$, Jung-Ah Hwang ${ }^{1}$, Jieun Lee ${ }^{1}$, \\ Il-Ju Choi ${ }^{4}$, Hyehyun $\mathrm{Seo}^{3}$, Jong-Hoon Park ${ }^{6}$, Hiromu Suzuki', Eiichiro Yamamoto ${ }^{7}$, \\ In-Hoo Kim ${ }^{5}$, Jin Sook Jeong ${ }^{8}$, Mi Ha Ju${ }^{8}$, Dong-Hee Lee ${ }^{9}$, and Yeon-Su Lee ${ }^{1}$ \\ ${ }^{1}$ Cancer Genomics Branch, Research Institute, National Cancer Center, Republic of Korea, \\ ${ }^{2}$ New Experimental Therapeutics Branch, Research Institute, National Cancer Center, Republic of Korea, \\ ${ }^{3}$ Genitourinary Cancer Branch, Research Institute, National Cancer Center, Republic of Korea, \\ ${ }^{4}$ Center for Gastric Cancer, Research Institute, National Cancer Center, Republic of Korea, \\ ${ }^{5}$ Molecular Imaging and Therapy Branch, Research Institute, National Cancer Center, Republic of Korea, \\ ${ }^{6}$ Department of Biological Science, Sookmyung Women's University, Seoul, Republic of Korea, \\ 7 Department of Molecular Biology, Sapporo Medical University, Sapporo, Japan, \\ ${ }^{8}$ Department of Pathology, Dong-A University College of Medicine, Busan, Republic of Korea, \\ ${ }^{9}$ Department of Life Science, Ewha Woman's University, Seoul, Republic of Korea \\ * These two authors contributed equally to this work. \\ Correspondence to: Yeon-Su Lee, email: yslee2@ncc.re.kr
}

Dong-Hee Lee, email: lee@ewha.ac.kr

Keywords: gastric cancer, adenylate cyclase, tumorigenesis, CAMP/PKA/CREB pathway, promoter methylation Received: August 23, $2013 \quad$ Accepted: September 28, $2013 \quad$ Published: September 30, 2013

This is an open-access article distributed under the terms of the Creative Commons Attribution License, which permits unrestricted use, distribution, and reproduction in any medium, provided the original author and source are credited.

ABSTRACT:

Adenylate cyclase $3(A D C Y 3)$ is a widely expressed membrane-associated protein in human tissues, which catalyzes the formation of cyclic adenosine-3', $5^{\prime}-$ monophosphate (CAMP). However, our transcriptome analysis of gastric cancer tissue samples (NCBI GEO GSE30727) revealed that ADCY3 expression was specifically altered in cancer samples. Here we investigated the tumor-promoting effects of ADCY3 overexpression and confirmed a significant correlation between the upregulation of $A D C Y 3$ and Lauren's intestinal-type gastric cancers. ADCY3 overexpression increased cell migration, invasion, proliferation, and clonogenicity in HEK293 cells; conversely, silencing ADCY3 expression in SNU-216 cells reduced these phenotypes. Interestingly, $A D C Y 3$ overexpression increased both the mRNA level and activity of matrix metalloproteinase 2 (MMP2) and MMP9 by increasing the levels of cAMP and phosphorylated CAMP-responsive element-binding protein (CREB). Consistent with these findings, treatment with a protein kinase A (PKA) inhibitor decreased MMP2 and MMP9 expression levels in ADCY3-overexpressing cells. Knockdown of ADCY3 expression by stable shRNA in human gastric cancer cells suppressed tumor growth in a tumor xenograft model. Thus, ADCY3 overexpression may exert its tumor-promoting effects via the CAMP/PKA/CREB pathway. Additionally, bisulfite sequencing of the $A D C Y 3$ promoter region revealed that gene expression was reduced by hypermethylation of CpG sites, and increased by 5-Aza-2'-deoxycytidine (5-AzadC)-induced demethylation. Our study is the first to report an association of ADCY3 with gastric cancer as well as its tumorigenic potentials. In addition, we demonstrate that the expression of $A D C Y 3$ is regulated through an epigenetic mechanism. Further study on the mechanism of $A D C Y 3$ in tumorigenesis will provide the basis as a new molecular target of gastric cancer. 


\section{INTRODUCTION}

Gastric cancer is the fourth most prevalent cancer type and the second largest contributor to cancer-related deaths worldwide. In developing countries, the incidence and mortality for gastric cancer are greater (2.58 and 3.06 times higher, respectively) than those in Western countries [1]. In Korea and Japan, high incidence rates of gastric cancer have prompted the initiation of national cancer screening programs [2]. These programs have led to the diagnosis and treatment of $50 \%$ more patients with early gastric cancer than was previously possible $[3,4]$, thereby contributing to decreased mortality [5]. However, many patients are not diagnosed until advanced stages, when metastasis to the lymph nodes or other organs has occurred [6], often rendering curative treatment impossible. Therefore, early diagnosis of gastric cancer remains an important issue, and the development of diagnostic tools such as molecular markers of gastric cancer is necessary to allow tumors to be distinguished from adjacent normal tissue.

Although a number of genes and pathways associated with gastric cancer have been identified by microarray analysis $[7,8]$, the molecular mechanisms underlying progression of the disease are not well understood. The present study was designed to identify novel genes involved in the development of early stage gastric cancer and to shed light on their roles in tumorigenesis. We performed microarray analyses to compare gene expression profiles of human gastric cancer samples and adjacent normal tissue (NCBI GEO accession no.: GSE30727) [9]. $A D C Y 3$ was one of the genes that were significantly overexpressed in gastric cancer samples, relative to the normal tissue.

Adenylate cyclases catalyze the formation of cyclic adenosine-3',5'-monophosphate (cAMP), the universal second messenger, from adenosine triphosphate (ATP). The adenylate cyclase family consists of 10 members, which show high primary sequence similarity at the catalytic site [10]. Gene regulation patterns and tissue distribution appear to be family member-specific, indicating these distinct functions are important in achieving precise regulation of cellular signals in a spatiotemporal manner. Consistent with this idea, knockout and transgenic mice models have revealed distinctive physiological roles for each adenylate cyclase family member [11]. The $A D C Y 3$ gene is located on chromosome 2p23.3 and contains 21 exons; the 4.41-kb mRNA transcript is translated into a $129-\mathrm{kDa}$ protein. The $A D C Y 3$ gene was initially identified in olfactory neuroepithelium [12] and in human islet cells [13]. ADCY3-knockout mice showed peripheral and behavioral anosmia [14], but to date, there has been no evidence linking $A D C Y 3$ to tumorigenesis.

In this study, we provide the first evidence that upregulation of $A D C Y 3$ in gastric cancer cells and tissues is associated with increased tumorigenic potential. We show that $A D C Y 3$ upregulation increases cell migration, invasiveness, proliferation, and clonogenicity, via the activation of CREB and its downstream pathways. In addition, DNA methylation analysis of the promoter region indicates that $A D C Y 3$ expression is regulated through an epigenetic mechanism.

\section{RESULTS}

\section{Gastric cancer-specific overexpression of $A D C Y 3$}

Comparison of gene expression profiles from microarray data revealed that $A D C Y 3$ was significantly upregulated in gastric cancer tissues $\left(P=4.215 \times 10^{-10}\right.$; Table 1). We validated this by using quantitative RT-PCR to measure $A D C Y 3$ expression levels in gastric cancer cells and tissues. $A D C Y 3$ mRNA levels in six human gastric cancer cell lines (SNU-216, SNU-638, SNU-719, AGS, KATO III, and MKN28) were significantly higher than those in normal cell lines (HDF, HMEC, and Hs738; Figure 1A). Tissue-specific $A D C Y 3$ expression at the mRNA level was examined in nine normal human tissues by RT-PCR. Heart, placenta, lung, and pancreas tissues showed high levels of $A D C Y 3$ expression, whereas brain, liver, and stomach tissues showed much lower levels of expression (Figure 1B). Such organ-specific expression may indicate distinct roles for $A D C Y 3$ in different tissues. Gastric cancer-specific overexpression of $A D C Y 3$ was confirmed in 14 of $21(66.7 \%)$ Korean gastric cancer tissues showing significantly higher $A D C Y 3$ mRNA levels than those in adjacent normal tissues. Significantly elevated $A D C Y 3$ expression was also observed in $80 \%$ of the Japanese gastric cancer cases analyzed (Figure 1C).

Because adenylate cyclase family members share significant sequence similarity, we examined their expression patterns in gastric cancer cell lines. When the gastric cancer cell lines (SNU-216, SNU-638, SNU719, AGS, KATO III, and MKN28) and the normal cell lines (HDF and HMEC) were subjected to RT-PCR using primers specific for each family member (Figure 1D), only $A D C Y 3$ showed a gastric cancer-specific expression pattern. $A D C Y 1$ expression was detected in both the normal cell lines, but in only one gastric cancer cell line (SNU-216). Expression of $A D C Y 2, A D C Y 4, A D C Y 5$, $A D C Y 8$, and $A D C Y 10$ was not detected in any of the cell lines, whereas expression of $A D C Y 6$ was consistently detected. Different levels of $A D C Y 7$ and $A D C Y 9$ were detected in gastric cancer and normal cell lines. These findings suggest that $A D C Y 3$ confers a tumorigenic effect on gastric cells, whereas the other members of the adenylate cyclase family do not. 
Table 1: The top 10 genes show a significant differential gene expression in microarray analysis using Benjamini and Hochberg False Discovery Rate (FDR).

\begin{tabular}{|c|c|c|c|c|}
\hline Gene symbol & Gene annotation & Chromosome & Position start No. & $P$-value \\
\hline$M S R I$ & macrophage scavenger receptor 1 & 8 & 15944161 & $7.530 \times 10^{-12}$ \\
\hline LOC44230 & $\begin{array}{l}\text { smilar to Interfer on-induced } \\
\text { transm em brane protein } 3 \text { (Interferon- } \\
\text { inducible protein 1-SU) }\end{array}$ & 11 & 305628 & $2.600 \times 10^{-10}$ \\
\hline CTSLI & cathep $\sin$ L 1 & 9 & 89530264 & $2.851 \times 10^{-10}$ \\
\hline ILS & interleukin 8 & 4 & 74825107 & $2.691 \times 10^{-10}$ \\
\hline SERPINHI & $\begin{array}{l}\text { serpin peptidase inhibitor clade } \mathrm{H} \\
\text { (heat shock protein } 47 \text { ) member } 1 \\
\text { (coll agen binding protein } 1 \text { ) }\end{array}$ & 11 & 74938382 & $2.975 \times 10^{-10}$ \\
\hline PTGS2 & $\begin{array}{l}\text { prostaglandin-endoper oxide synthase } \\
2 \text { (prostaglandin } \mathrm{G} / \mathrm{H} \text { synthase and } \\
\text { cyclooxygenase) }\end{array}$ & 1 & 184907556 & $4.964 \times 10^{-10}$ \\
\hline$L Y 6 E$ & $\begin{array}{l}\text { lymphocyte antigen } 6 \text { complex locus } \\
\text { E }\end{array}$ & 8 & 144148702 & $4.281 \times 10^{-10}$ \\
\hline$A D C Y 3$ & adenylate cyclase 3 & 2 & 24895545 & $4.215 \times 10^{-10}$ \\
\hline$T U L P 3$ & tubby like protein 3 & 12 & 2938471 & $4.981 \times 10^{-10}$ \\
\hline HTRAI & Htr A serine peptidase 1 & 10 & 124211033 & $4.485 \times 10^{-10}$ \\
\hline
\end{tabular}

A

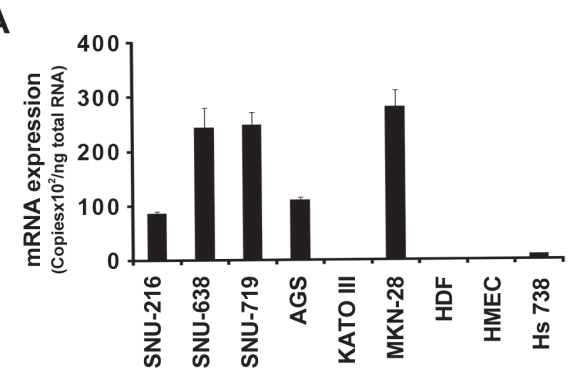

C

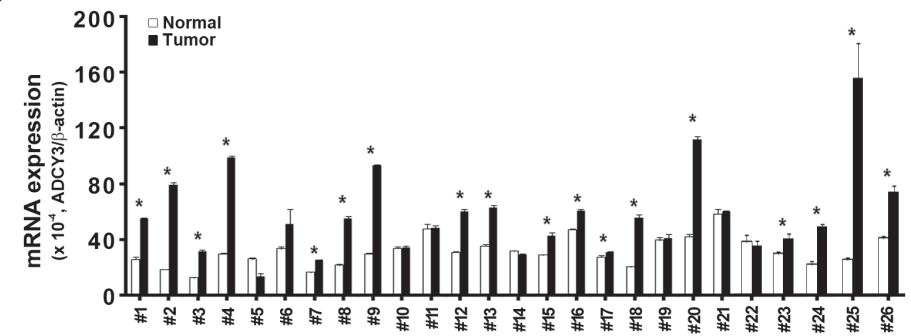

D

B

ADCY3

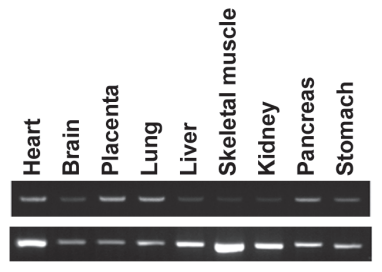

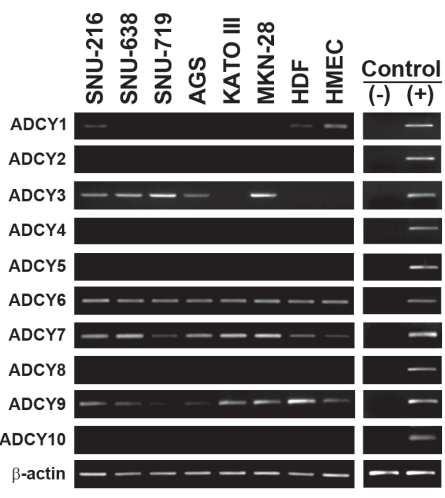

Figure 1: $A D C Y 3$ mRNA expression levels in gastric cancer cells and tissues. (A) Absolute quantification of $A D C Y 3$ mRNA in gastric cancer cells (SNU-216, SNU-638, SNU-719, AGS, KATOIII, MKN28) and normal human cells (HDF, HMEC, Hs738). (B) $A D C Y 3$ mRNA expression in various human tissues. Nine tissue samples, including heart, brain, placenta, lung, liver, skeletal muscle, kidney, pancreas, and stomach samples (Multiple-tissue cDNA panel, Clontech) were amplified. $A D C Y 3$ expression differed in various tissues. (C) Relative quantification of $A D C Y 3$ mRNA in gastric cancer tissues and adjacent normal tissues of Korean (\#1-\#21) and Japanese patients (\#22-\#26), * indicates $P<0.05$ between gastric cancer vs. adjacent normal tissues. Blank and solid columns indicate normal and cancer tissues, respectively. Error bars indicate standard deviations. (D) Expression specificity of ADCY family members detected using RT-PCR in 6 gastric cancer cell lines (SNU-216, SNU-638, SNU-719, AGS, KATOIII, and MKN28) and 2 normal cells (HDF and HMEC). Among the 10 family members of $A D C Y \mathrm{~s}, A D C Y 6$ appeared in all cell lines tested in this analysis. $A D C Y 1, A D C Y 3$, and $A D C Y 7$ showed cell line-dependent expression; $A D C Y 3$ showed expression only in gastric cancer cell lines, but not in normal cell lines. (-) indicates the non-template negative control. (+) indicates the positive controls. The U2OS cell line cDNA was used for $A D C Y 1,2,4,5,10$, and U87 cell line cDNA was used for $A D C Y 3,6,7,8$, and 9. Beta-actin was used as a loading control. 


\section{Clinicopathological properties of $A D C Y 3$ in gastric cancer}

Immunohistochemical analysis showed that $A D C Y 3$ was not expressed in non-neoplastic gastric surface and foveolar epithelial cells (Figure 2). Immunohistochemical analysis of 203 patient samples revealed 126 (62.1\%) cases with a level of $A D C Y 3$ protein expression that was consistent with the mRNA level measured by qRT-PCR. When we categorized $A D C Y 3$ signal intensities into three levels (mild, moderate, or strong), we observed a significant association of higher $A D C Y 3$ expression levels with the differentiated histologic subtype $(P<$ 0.0001; Table 2A), as well as with Lauren's intestinal type $(P<0.0001$, Table 2B). There was no significant association between $A D C Y 3$ overexpression and other clinicopathological parameters, including, age, sex, depth of invasion, vascular invasion, or TNM staging.

\section{Role of $A D C Y 3$ in gastric cancer tumorigenesis}

To examine the function of $A D C Y 3$ in gastric cancer development, we investigated the effects of $A D C Y 3$ overexpression or downregulation on tumorigenesisrelated cellular characteristics such as cell migration, invasiveness, cell viability, and clonogenicity. For HEK293 cells transfected with pAcGFP-ADCY3 (Figure $\mathrm{S} 1 \mathrm{~A}$ ), the number of migrating cells significantly increased by approximately $43 \%(P=0.01$; Figure $3 \mathrm{~A})$.
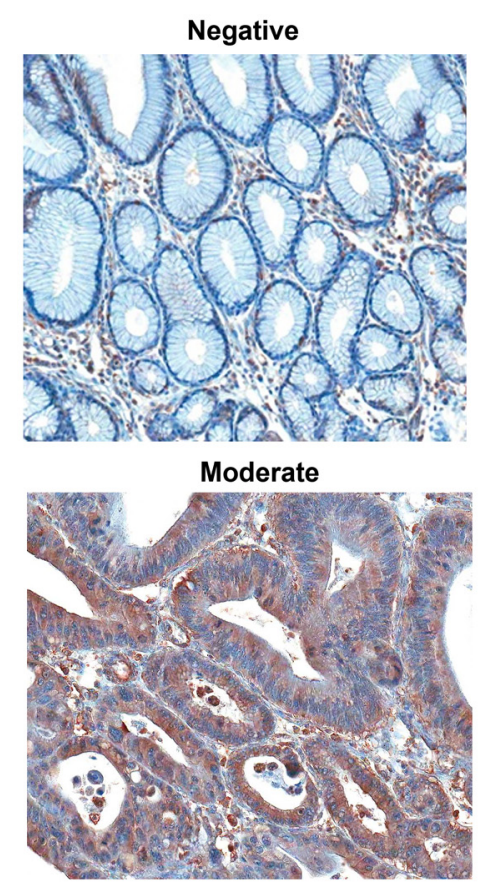

Consistent with this finding, $A D C Y 3$-overexpressing cells showed a 4.95 -fold increase in invasiveness $(P$ $=0.02)$ compared to cells transfected with the control vector (Figure 3B). Moreover, in MTT proliferation assays, pAcGFP- $A D C Y 3$-transfected cells showed higher viability than did the control cells $(P<0.001$; Figure $3 C)$. Clonogenic assays also revealed a reinforcement effect on anchorage-dependent cell growth $(P<0.005)$ in pAcGFP$A D C Y 3$-overexpressing cells (Figure $3 \mathrm{D}$ ). In contrast, SNU-216 gastric cancer cells treated with $A D C Y 3$-specific siRNA showed a loss of tumorigenesis-related cellular characteristics. $A D C Y 3$ downregulation caused by siRNA (Figure S1B) decreased cell migration by $21 \%(P=0.018$; Figure 3E), cell invasiveness by 5.29 -fold $(P=0.016$; Figure $3 \mathrm{~F})$, cell viability by $12 \%(P<0.01)$ following a 48-hr siRNA treatment (Figure 3G), and clonogenicity by 1.8 -fold ( $P=0.002$; Figure $3 \mathrm{H})$. These results collectively indicate that $A D C Y 3$ functions during tumorigenesis of gastric cancer by changing cellular characteristics toward cancer cells.

\section{ADCY3 overexpression activates CREB and upregulates $M M P 2$ and $M M P 9$}

To investigate the molecular pathway of $A D C Y 3$ in gastric cancer tumorigenesis, we analyzed the functional consequences of $A D C Y 3$ overexpression. First, we measured cAMP levels in pAcGFP- $A D C Y 3$-transfected HEK293 cells, because $A D C Y 3$ is known to catalyze cAMP formation. In cells overexpressing $A D C Y 3$, we

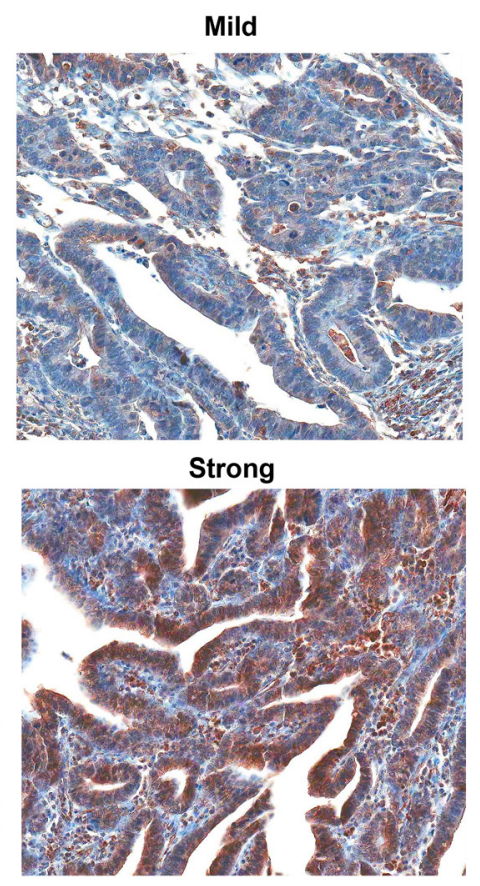

Figure 2: Immunohistochemical detection of $A D C Y 3$ expression in normal and non-neoplastic gastric mucosal glands and gastric carcinomas (magnification 200x). Non-neoplastic foveolar epithelial cells did not show immunostaining. Gastric adenocarcinomas represent $A D C Y 3$ expression with mild, moderate or strong immunostaining. Subcellular location of $A D C Y 3$ expression was mostly cytoplasmic. 
Table 2: Correl ation between $A D C Y 3$ expression and differentiation of gastric carcinomas.

A. Correlation of $A D C Y 3$ expression to differentiation grade of tubular adenocarcinoma and signet ring cell carcinoma

\begin{tabular}{|c|c|c|c|c|c|}
\hline \multirow{3}{*}{ Histologic type } & \multicolumn{5}{|c|}{ ADCY3 Expression } \\
\hline & \multirow{2}{*}{ Negative (\%) } & \multicolumn{3}{|c|}{ Positive } & \multirow{2}{*}{$\mathrm{N}$} \\
\hline & & Mild (\%) & Moderate (\%) & Strong (\%) & \\
\hline $\begin{array}{l}\text { Tubular adenocarcinoma, } \\
\text { well differentiated }\end{array}$ & $8(17.0)$ & $9(19.2)$ & $25(53.2)$ & $5(10.6)$ & 47 \\
\hline $\begin{array}{l}\text { Tubular adenocarcinoma, } \\
\text { moder ately differentiated }\end{array}$ & $12(21.4)$ & $29(51.8)$ & $12(21.4)$ & $3(5.4)$ & 56 \\
\hline $\begin{array}{l}\text { Tubular adenocarcinoma, } \\
\text { poorly differentiated }\end{array}$ & $44(53.6)$ & $24(29.3)$ & $11(13.4)$ & $3(3.7)$ & 82 \\
\hline Signet ring cell carcinoma & $13(72.2)$ & $5(27.8)$ & $0(0)$ & $0(0)$ & 18 \\
\hline $\mathrm{N}$ & 77 & 67 & 48 & 11 & 203 \\
\hline
\end{tabular}

$X^{2}$-test 58.742 ; DF: 9 ; Significance level: $p<0.0001$; Contingency coefficient 0.474

B. Correlation of $A D C Y 3$ expression to subtypes of gastric carcinoma by Lauren's classification

\begin{tabular}{|c|c|c|c|c|c|}
\hline \multirow{3}{*}{$\begin{array}{l}\text { Lauren } \\
\text { classification }\end{array}$} & \multicolumn{5}{|c|}{$\mathrm{ADCY} 3$ Expression } \\
\hline & \multirow{2}{*}{ Negative (\%) } & \multicolumn{3}{|c|}{ Positive } & \multirow{2}{*}{$\mathrm{N}$} \\
\hline & & Mild (\%) & Moderate (\%) & Strong (\%) & \\
\hline Intestinal & $17(18.7)$ & $31(34.1)$ & $36(39.5)$ & $7(7.7)$ & 91 \\
\hline Mixed & $14(45.2)$ & $13(41.9)$ & $3(9.7)$ & $1(3.2)$ & 31 \\
\hline Diffuse & $46(56.8)$ & $23(28.4)$ & $9(11.1)$ & $3(3.7)$ & 81 \\
\hline $\mathrm{N}$ & 77 & 67 & 48 & 11 & 203 \\
\hline
\end{tabular}

$X^{2}$-test 37.484 ; DF: 6 ; Significance level: $p<0.0001$; Contingency coefficient 0.395 
A

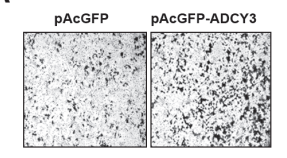

C

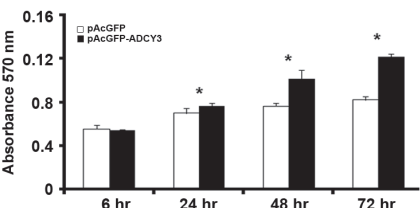

E

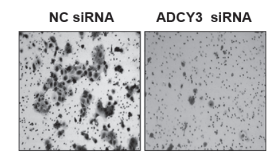

G

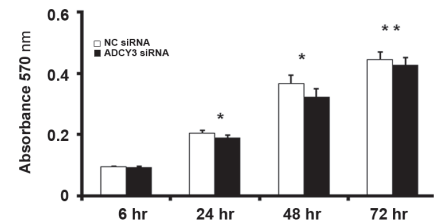

B

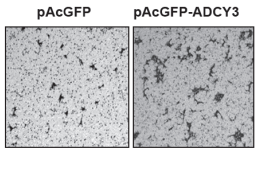

D
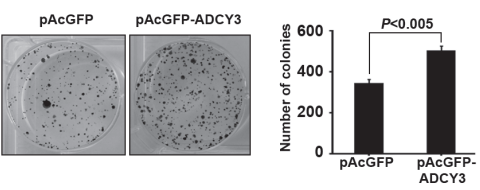

F
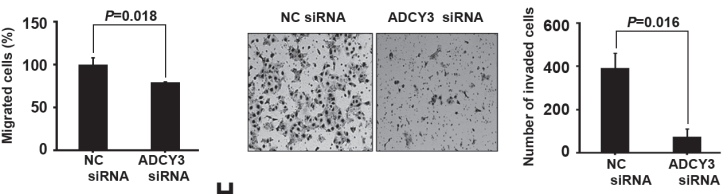

H
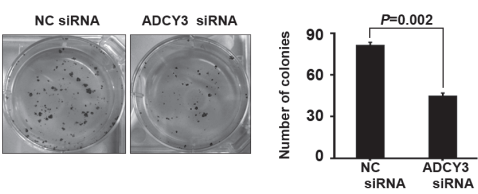

Figure 3: Effect of $A D C Y 3$ expression on tumorigenesis-related cell behaviors. Increment of migration, invasiveness, and colony formation in ADCY3 overexpressing HEK293 cells (A-D). (A) Cell migration determined using a Boyden transwell chamber. Cell migration was increased by $A D C Y 3$ overexpression. (B) Cell invasiveness was assayed in a Matrigel-coated transwell chamber. The number of cells crossing the Matrigel-coated filter was increased by $A D C Y 3$ overexpression. (C) Cell growth of control vector cells and $A D C Y 3$ overexpressing cells were examined using an MTT assay. * indicates $P<0.0001$ between $A D C Y 3$ expressing plasmid vs. control vector (D) Clonogenicity of the HEK293 cells transfected with pAcGFP-ADCY3 showed an increase. Determination of the effects of $A D C Y 3$ silencing on cell migration, invasiveness, and colony formation by siRNA treatment on SNU-216 gastric cancer cell line (E-H). Cell migration (E), invasiveness (F), MTT assay $(\mathrm{G})$, and clonogenicity $(\mathrm{H})$ of SNU-216 cells following ADCY3-specific siRNA treatment was decreased compared with that by negative control (NC) siRNA.

A

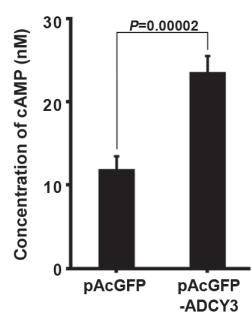

D

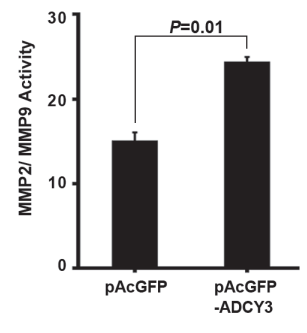

B

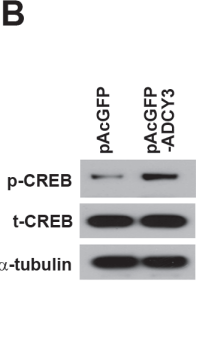

C

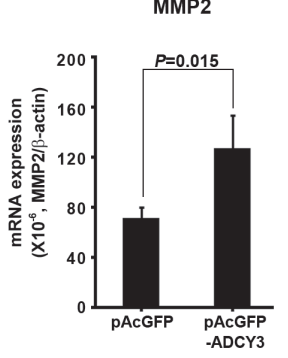

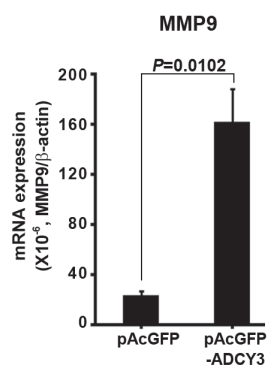

MMP9

E
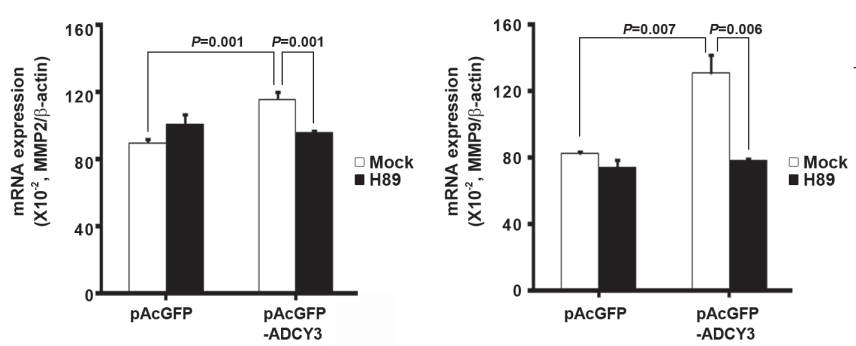

Figure 4: The molecular mechanism of $A D C Y 3$ in gastric cancer tumorigenesis. (A) Determination of cAMP concentration increment following forced ADCY3 expression in HEK293 cells. The level of cAMP of ADCY3 overexpressing cells showed a twofold increase compared with the blank vector control. (B) Levels of phosphorylated CREB (p-CREB) at Ser133 were increased following $A D C Y 3$ overexpression compared to the consistent level of total-CREB (t-CREB) between blank vector control and pAcGFP-ADCY3 overexpression. Alpha-tubulin was used as a loading control. The mRNA expression level (C) and activities (D) of MMP2 and MMP9 measured in pAcGFP-ADCY3-transfected cells were significantly increased compared to the blank vector control. (E) After PKA inhibitor treatment, mRNA expression of $M M P 2$ and $M M P 9$ was decreased in $A D C Y 3$ overexpressing cells. 
observed a 1.99-fold increase in cAMP concentration ( $P=$ 0.00002 ) compared to the control, indicating that $A D C Y 3$ overexpression affects cAMP formation (Figure 4A). We further investigated the effects of cAMP accumulation on the progression of gastric cancer by measuring CREB activity by comparing the amounts of total CREB (t-CREB) and phosphorylated CREB (p-CREB) at the Ser133 residue. Consistent with the changes in cAMP levels that we observed, the levels of p-CREB was significantly higher in pAcGFP-ADCY3-transfected cells than that in control cells, whereas the level of t-CREB remained unchanged (Figure 4B). To further understand the effect of increased p-CREB on downstream pathways, we investigated the effect of changes in $A D C Y 3$ levels on $M M P 2$ and $M M P 9$ expression and activity. $M M P 2$ and $M M P 9$ are activated by CREB and appear to play important roles in tumor invasion and metastasis $[15,16]$. In pAcGFP-ADCY3-transfected cells, in which cAMP and p-CREB were elevated, the mRNA levels of $M M P 2$ and $M M P 9$ were also significantly increased (Figure 4C). We confirmed these results by measuring the activity of $M M P 2$ and $M M P 9$ in $A D C Y 3$-overexpressing cells; as expected, significantly increased $M M P 2 / M M P 9$ activity was observed $(P=0.01$; Figure $4 \mathrm{D})$. Next, we examined whether the observed increase in $M M P 2$ and $M M P 9$ mRNA levels was specifically due to cAMP/PKA/ CREB-mediated pathway activation. We therefore treated $A D C Y 3$-transfected cells with a PKA inhibitor (H89) and found that it decreased $M M P 2$ and $M M P 9$ expression levels $(P=0.001$ and $P=0.006$, respectively; Figure $4 \mathrm{E})$. These results suggest that $A D C Y 3$ promotes gastric cancer development via activation of cAMP-mediated CREB signaling, and upregulation of $M M P 2$ and $M M P 9$ expression.

A

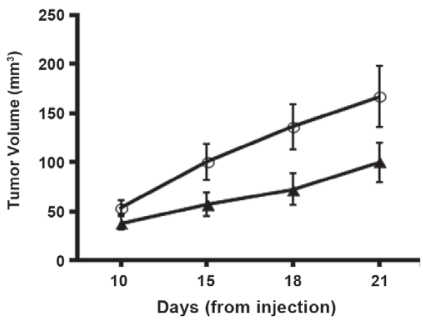

B

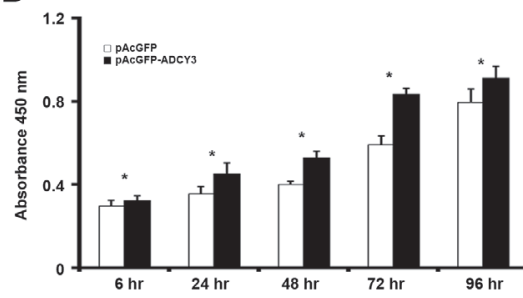

$A D C Y 3$ knockdown suppresses tumor growth and cell proliferation

To investigate the role of $A D C Y 3$ in tumorigenesis, we used the tumor xenograft model by implanting established gastric cancer cells (MKN28) with or without $A D C Y 3$ knockdown. We confirmed that the level of ADCY3 protein was highly decreased in ADCY3knockdown MKN28 cells before implantation (Figure S1C). Nude mice were subcutaneously injected with $A D C Y 3$-knockdown or pLL3.7 control cells, and tumor volumes were measured three weeks after injection. Tumor volumes in mice injected with $A D C Y 3$-knockdown MKN28 cells were significantly lower than those in mice injected with control pLL3.7-transfected cells (Figure 5A). This result suggests that stable knockdown of $A D C Y 3$ in gastric cancer cells can suppress tumor growth.

Next, to examine the effect of $A D C Y 3$ expression on cell growth, we used a bromo-deoxyuridine (BrdU) incorporation assay to measure cell proliferation. The proliferation of $A D C Y 3$-overexpressing cells was significantly higher than that of control cells $(P<0.01$; Figure 5B). Conversely, the proliferation of $A D C Y 3$ knockdown cells was significantly lower than that of control cells $(P<0.01$; Figure 5C).

\section{Epigenetic regulation of $A D C Y 3$ expression in cancer cells}

The role of DNA methylation in the regulation of cancer-related gene expression has been well examined. We therefore investigated whether $A D C Y 3$ expression is

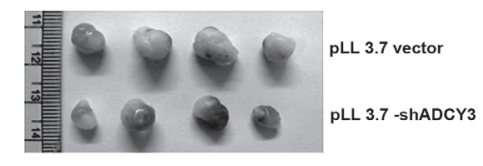

C

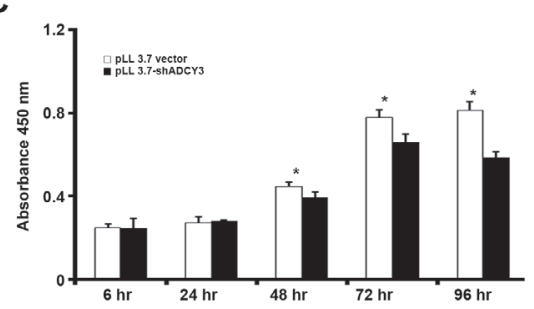

Figure 5: Suppression of tumor growth and cell proliferation. (A) pLL 3.7 vector or pLL 3.7-sh $A D C Y 3$ transfectants were injected subcutaneously into nude mice. Tumor growth curve (left panel) and tumor images (right panel). Cell proliferation was assessed using the BrdU incorporation assay in ADCY3 overexpressing HEK293 cells (B) and ADCY3 knockdown MKN28 cells (C). Cells were incubated with BrdU for $4 \mathrm{hr}$, and labeled cells were measured at absorbance $450 \mathrm{~nm}$. The first absorbance measurement was performed at $6 \mathrm{hrs}$ after cell plating for firm cell attachment. Further measurements were done at every 24 hrs after cell plating until 72 hrs. 
regulated by $\mathrm{CpG}$ methylation in the promoter region. To screen for $\mathrm{CpG}$ islands in the $A D C Y 3$ promoter, we performed methylation-sensitive high-resolution melting (MS-HRM) analysis on a 600-bp region immediately upstream of the translational start site (ATG codon). We designed three sets of primer pairs to amplify PCR products spanning the 457-bp region from 585 to 129 bp upstream of the translational start site (Figure 6A). However, we did not observe clear differences in methylation levels when six different gastric cancer cell lines were analyzed (Figure 6B). MS-HRM primers could not be designed for the region 694-387 bp upstream of the translational start site, where a 308-bp CpG island was located, due to the high density of $\mathrm{CpG}$ sites. Instead, this region was analyzed by cloning and sequencing of bisulfite-treated DNA. We observed hypomethylation in cell lines such as SNU-216, SNU-638, SNU-719, AGS, and MKN28, in which $A D C Y 3$ is highly expressed.

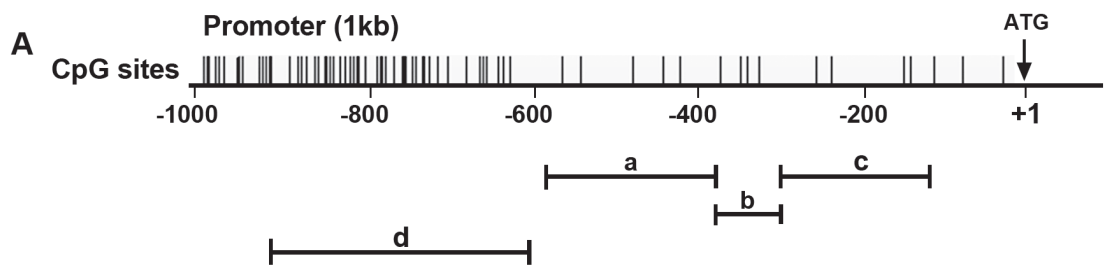

B
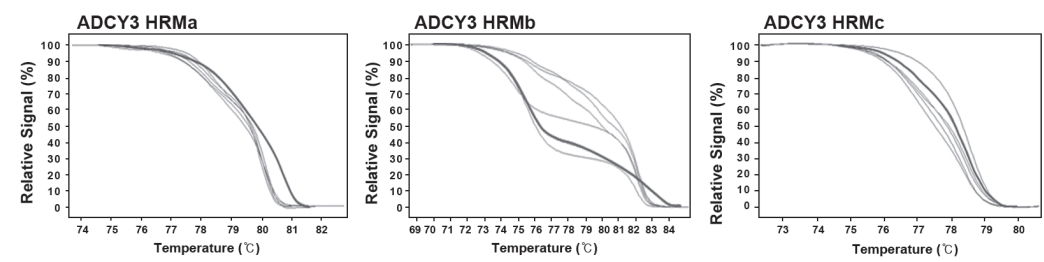

C

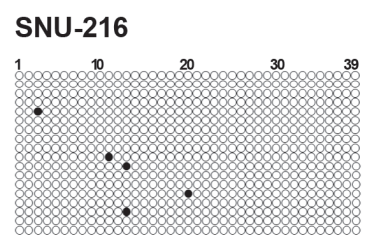

SNU-638

SNU-719
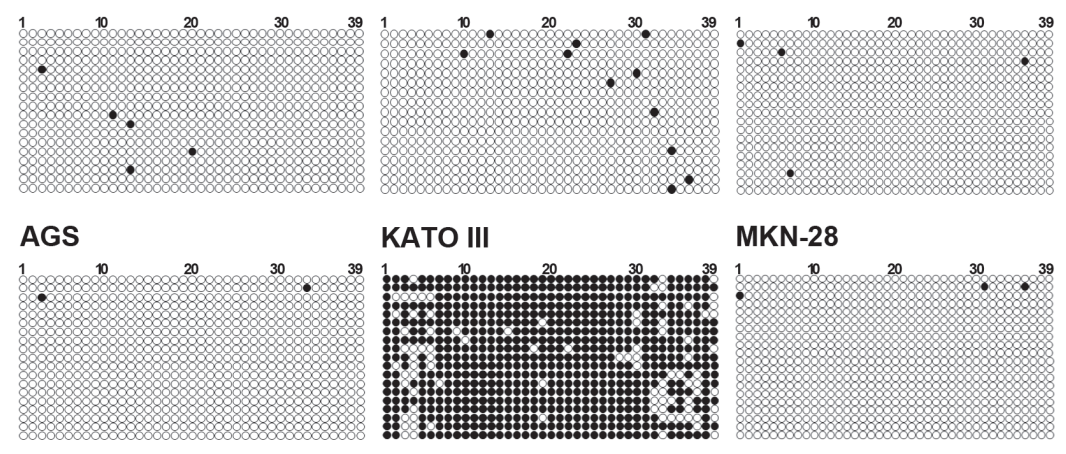

KATO III

MKN-28
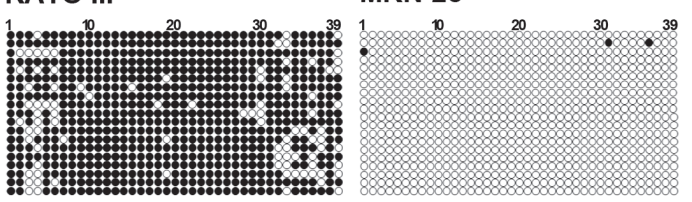

D

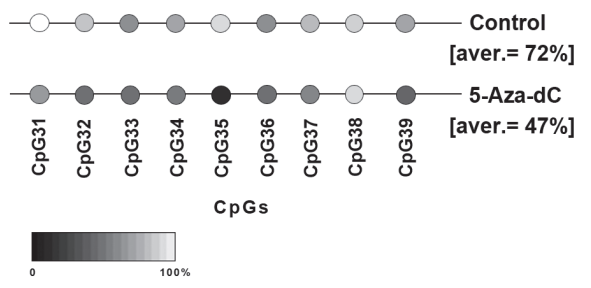

E

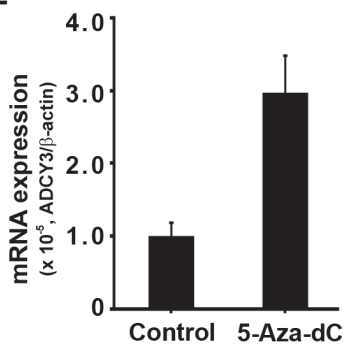

Figure 6: Regulation of $A D C Y 3$ mRNA expression by DNA methylation. (A) DNA methylation status of $A D C Y 3$ in gastric cancer cell lines analyzed by bisulfite sequencing. Top, map of the $\mathrm{CpG}$ island. Individual $\mathrm{CpG}$ dinucleotides are indicated as vertical lines. ( $a, b$, and c; region of MS-HRM screening, d; region of bisulfite sequencing) Bottom, each circle indicates a CpG site in the promoter sequence, and horizontal lines indicate the sequence of the individual clone. White and black circles depict unmethylated and methylated CpG sites, respectively. (B) Results of methylation-sensitive high-resolution melting (MS-HRM) analysis. These regions did not show significant differences in methylation status between cancer and normal cell lines. (C) Results of bisulfite sequencing. A total of 6 cell lines were surveyed for their methylation status by bisulfite sequencing, and only the KATO-III cell line showed significant methylation on region d of panel A. The demethylation by 5-Aza-dC treatment reduced $A D C Y 3$ promoter methylation compared with negative control (D) and also restored ADCY3 expression in the KATOIII cell line according to qRT-PCR results. Control represents no 5-Aza-dC treatment (E). 
Conversely, hypermethylation was observed in the $A D C Y 3$-non-expressing KATO III cell line (Figure 6C). Furthermore, treatment of the KATO III cell line with 5-Aza-2'-deoxycytidine (5-Aza-dC), a demethylating agent, resulted in demethylation of $\mathrm{CpG}$ sites in the $A D C Y 3$ promoter (Figure 6D) and upregulation of $A D C Y 3$ expression (Figure 6E). Taken together, these results suggest that DNA methylation of $\mathrm{CpG}$ islands in the promoter region indeed regulates $A D C Y 3$ expression.

\section{DISCUSSION}

Gastric cancer is a complex disease caused by the interaction of diverse factors [17]. Although novel genetic variations associated with gastric cancer have recently been identified using genome-wide screening $[18,19]$, the detailed molecular mechanisms remain largely unknown. Couple of current studies addressed the candidates of new molecular targets for gastric cancer, including MET amplification [20] and a epigenetically regulated tumor-suppressor, galecin-7 [21]. However, additional studies will be necessary to completely define the genetic alterations underlying tumorigenesis in gastric cancer [22] for the development of new therapeutics. Our gene expression profiling study compared cancerous and adjacent normal tissue samples from 27 Korean gastric cancer patients, and identified $A D C Y 3$ as a candidate gastric cancer gene. We validated gastric cancer-specific upregulation of $A D C Y 3$ by using two different methods, qRT-PCR and Immunohistochemical analysis, in 229 gastric cancer samples from patients in three different groups. We also found evidence for a functional association between $A D C Y 3$ and gastric cancer, through CREB pathway-dependent effects on cell migration, invasiveness, proliferation, and clonogenicity. Lastly, our data also support an epigenetic mechanism for the regulation of $A D C Y 3$ expression.

Another adenylate cyclase family member, $A D C Y 2$, was recently reported to be associated with altered survival in colorectal cancer [23]. This finding suggests that tissuespecific alternation of $A D C Y$ family members in human tissues contributes to the onset of tissue-specific diseases such as cancer. Consistent with our finding of tissuespecific $A D C Y 3$ expression levels (Figure 1B), a survey of gene-set enrichment databases at the Broad Institute (http:/www.broadinstitute.org/GSEA) revealed a highly specific expression pattern of $A D C Y 3$ in human tissues, notably upregulation in placenta, as well as in MOLT4 leukemic cells, HL-60 cells, and B lymphoblasts.

Deregulation of the effector molecule cAMP is known to be involved in tumorigenesis [24], and reduction in cAMP has an anti-proliferative effect in colorectal cancer cells [25]. The effects of cAMP are mainly mediated by protein kinase A (PKA) [26]. cAMP/protein kinase A (PKA) signaling, the most prominent pathway downstream of the cAMP generated by ADCYs, is the primary mechanism responsible for phosphorylation of CREB [27]. Growing evidence implicates CREB as a proto-oncogene in many types of cancer [28]. In human melanoma cells, CREB has been shown to be a mediator of tumorigenesis and metastasis [29]. CREB-binding proteins are also involved in the development of Ewing sarcoma [30]. In addition, as reported for breast cancer [31], cAMP/PKA signaling regulates actin-based cell migration through cytoskeletal remodeling. Our findings suggest that the elevated cAMP levels in gastric cancer cells generated by $A D C Y 3$ leads to increased tumor aggressiveness, as indicated by changes in properties such as cell migration, proliferation, and invasion. In our study, $A D C Y 3$ overexpression caused a considerable increase in the mobility, invasiveness, proliferation, and clonogenicity of cancer cells, whereas silencing of ADCY3 suppressed these activities. It has been reported that upregulation of phosphorylated CREB plays a role in the development of several cancers [27]. In particular, a correlation between phosphorylated CREB levels and tumor grading has been observed in pituitary adenoma [32], as well as in lung and prostate cancers [33]. Additionally, CREB is known to play essential roles in regulating $M M P 2$ and $M M P 9$ expression in melanoma [29] and pancreatic cancer cells [34]. The fact that the $M M P 2$ promoter contains a cAMP response element (CRE), at position -374 to -366 with respect to the transcription initiation site [35], supports a relationship between $A D C Y 3$ overexpression and enhanced tumorigenic cell behavior. In summary, our results indicate that elevated $A D C Y 3$ expression contributes to gastric cancer progression through the cAMP/PKA/CREB pathway, by increasing both mRNA expression and $M M P 2$ and MMP9 activity. These changes were blocked by PKA inhibitor treatment. Therefore, upregulated $A D C Y 3$ may lead to increased cell migration, invasion, and proliferation, which are characteristic of cancer. We used knockdown experiments to confirm the tumor-promoting effects of $A D C Y 3$ overexpression. $A D C Y 3$ knockdown reduced cell proliferation in vitro and suppressed tumor growth in a xenograft tumor model in vivo.

In gastric cancer, the tumorigenic potential of some proto-oncogene accounts for the amplification in cancer, such as MET gene amplification [20]. On the other hand, expression level of many oncogenes may be attributed by promoter activity that is subjected to epigenetic regulation as galectin-7 gene [21]. ADCY3 expression may be affected by promoter $\mathrm{CpG}$ methylation, a wellknown epigenetic mechanism for cancer-specific changes in gene expression. According to the encyclopedia of DNA elements (ENCODE) annotation [36], the region of the $A D C Y 3$ promoter analyzed in our study harbors binding sites for critical transcription factors, including early growth response protein 1 (Egr-1), E2F, and activating protein 2 (AP-2), which are known to promote the development of an environment conducive for tumor formation $[37,38]$. It is possible that environmental factors 
alter the methylation status of the $A D C Y 3$ promoter region, upregulating the expression of $A D C Y 3$ in gastric tissue. This could lead to activation of the CREB pathway, thereby promoting tumorigenesis.

This study is the first to report that $A D C Y 3$ is involved in cancer development. We found that $A D C Y 3$ is specifically upregulated in gastric cancer cell lines and tissues, and that overexpression of $A D C Y 3$ promotes tumorigenesis by increasing cell migration, proliferation, and invasiveness, through its effects on the CREB pathway. Results from a xenograft model also indicate that $A D C Y 3$ plays a crucial role in tumor growth. As more data on selective inhibitors for individual adenylate cyclase family members will be available [11], the development of $A D C Y 3$-based targeted therapeutic agents for gastric cancer may become possible. In summary, although further functional and clinical validation will be necessary, our results indicate that $A D C Y 3$ expression could be used as a prognostic marker for gastric cancer.

\section{MATERIALS AND METHODS}

\section{Cell culture and tissue samples}

The following cell lines were used in our study: human cancer cell lines SNU-216, SNU-638, and SNU719 (Korea Cell Line Bank, Seoul, Korea); AGS, KATO III, MKN28, H23, A549, HCT-116, SNU-81, SK-BR-3, JIMT-1, U2OS, and U87 (ATCC, Manassas, VA, USA); human dermal fibroblast (HDF; ATCC), normal human gastric (Hs783, ATCC), and HEK293 (ATCC). Cells were cultured in appropriate growth media (Corning Cellgro, Manassas, VA, USA) supplemented with $10 \%$ (v/v) fetal bovine serum and 1x penicillin-streptomycin (Invitrogen, Carlsbad, CA, USA). Human mammary epithelial cells (HMEC; Lonza, Basel, Switzerland) were cultured according to the manufacturer's instructions. Normal and gastric cancer tissue samples (Table S1) were obtained in accordance with the principles of the Declaration of Helsinki and were approved by the institutional review board of the National Cancer Center, Korea, the Akita Red Cross Hospital, and the Sapporo Medical University, Japan, for human subject studies.

\section{Detection of mRNA expression by RT-PCR and quantitative RT-PCR}

cDNA was synthesized from $2 \mu \mathrm{g}$ of random primed total RNA from cultured cells using SuperScript III FirstStrand Synthesis kit (Invitrogen). We obtained human cDNA from the Human Multiple Tissue cDNA panel I and Human Digestive System MTC panel (Clontech, Mountain View, CA, USA). Primers for RT-PCR or qRT-PCR were designed using Primer3 software (http://frodo.wi.mit. edu/primer3/) to span 2 consecutive exons of each gene (Table S2A). qRT-PCR reactions were performed using a LightCycler 480 (Roche Applied Science, Mannheim, Germany); results were quantified either by using $\beta$-actin for normalization (relative quantification), or by standard curve-based absolute quantification.

\section{Immunohistochemistry of gastric carcinoma tissues}

Immunohistochemistry (IHC) of $A D C Y 3$ was performed on 3 core cancer tissues ( $2 \mathrm{~mm}$ in diameter), including non-neoplastic gastric mucosal tissue from each patient. These were arranged as a tissue array of 4to $6-\mu \mathrm{m}$ sections mounted on silicon-coated glass slides. For detection, we used an anti-ADCY3 antibody (sc-588; Santa Cruz Biotechnology, Santa Cruz, CA, USA) with the BenchMark XT automated system (Ventana Medical System, Tucson, AZ, USA), as described previously [39]. Samples were scored as negative or positive based on the staining intensity and distribution of gastric carcinoma cells; positive cases were sub-categorized into mild, moderate, or strong expression groups. We screened a total of 203 patients who had undergone gastrectomy at the Dong-A University Hospital, Busan, South Korea from January 1, 2006 through December 31, 2006. Gastric carcinomas consisted of 47 well-differentiated, 56 moderately differentiated, 82 poorly differentiated, and 18 signet ring cell adenocarcinomas. Based on Lauren's classification, there were 91 intestinal type cases, 81 diffuse type cases, and 31 mixed type cases. No patients received any preoperative treatments.

\section{Assessment of $A D C Y 3$-dependent tumorigenic cell behavior and related pathways}

To overexpress $A D C Y 3$, HEK293 cells were transfected with pAcGFP1-ADCY3, using Lipofectamine 2000 (Invitrogen), with the empty pAcGFP-C1 vector used as a negative control. To silence the $A D C Y 3$ gene, $5 \times 10^{4}$ SNU-216 human gastric cancer cells were transfected with human $A D C Y 3$-specific small interfering RNA (siRNA) (siADCY3: SI00058849 5'-ATGGAGCACCAGCTTCCTCAA-3') or negative control (NC) siRNA (Cat. No. 1027280; Qiagen, Hilden, Germany) in a 6-well culture plate by using HiPerfect (Qiagen).

Cell migration was assayed by staining cells that had migrated downward through an $8-\mu \mathrm{m}$ pore filter insert (BD Biosciences, Franklin Lakes, NJ, USA) during an overnight incubation at $37^{\circ} \mathrm{C}$. Crystal violet $(1 \%)$ was used for cell staining, and $\mathrm{A}_{564}$ measurements were made using a VERSAmax microplate reader (Molecular Devices, Sunnyvale, CA, USA). Invasion assays were performed by counting cells stained Diff-Quick (Sysmex, 
Kobe, Japan) in Matrigel-coated inserts after $48 \mathrm{hr}$ of incubation. Cell viability was measured using an MTT assay. Briefly, cells were seeded in 96-well plates at 1,000 cells per well and incubated for $6 \mathrm{hr}$ to allow attachment, before measurements were made. Quantification of viable cells was based on absorbance measurements at $570 \mathrm{~nm}$, taken over the period from 6 to $72 \mathrm{hr}$ after cell seeding. Clonogenicity was assayed by seeding $1 \times 10^{3}$ cells per well in 6-well plates and counting the surviving colonies that were stained using Diff-Quick, after a 7-day incubation.

\section{Molecular assays of $A D C Y 3$ function in gastric cancer}

To confirm the function of expression of $A D C Y 3$ and its family members, we measured cAMP level increments depending on $A D C Y 3$ expression. HEK293 cells were transfected with $400 \mathrm{ng}$ of pAcGFP or pAcGFP-ADCY3 by using Lipofectamine 2000, and seeded in 96-well plates at a density of $2 \times 10^{4}$ cells/well. The cAMP levels in $50 \mu \mathrm{L}$ of cell lysate were measured using the CatchPoint cyclicAMP Fluorescent Assay Kit (Cat\# R8088, Molecular Devices) according to the manufacturer's protocol.

To measure protein expression of $A D C Y 3$, total CREB, and p-CREB, western blotting was performed using anti-ADCY3 (ab14778; Abcam, Cambridge, UK), anti-GFP (sc-9996, Santa Cruz Biotechnology), and antiCREB and anti-p-CREB Ser133 (\#8212, Cell Signaling Technology, Danvers, MA, USA); anti- $\beta$-actin and anti- $\alpha-$ tubulin (Sigma-Aldrich, St. Louis, MO, USA) antibodies were also used.

To quantify $M M P 2$ and $M M P 9$ activity, pAcGFP or pAcGFP-ADCY3 transfected cell lysate was diluted 1:3 with activation buffer, and assayed using the Gelatinase (MMP2/MMP9) activity assay kit (Millipore, Billerica, MA, USA), following the manufacturer's protocol. For experiments involving the PKA inhibitor, pAcGFP or pAcGFP-ADCY3 transfected cells $\left(3 \times 10^{5}\right)$ were treated with $20 \mu \mathrm{M}$ PKA inhibitor H89 (Sigma Aldrich) and incubated for $1 \mathrm{hr}$ at $37^{\circ} \mathrm{C}$.

\section{Tumor xenograft model}

A lentiviral vector ( $\mathrm{pLL3}$.7) was used for production of $A D C Y 3$ shRNA. Tumors were established by subcutaneously injecting $5 \times 10^{6} \mathrm{MKN} 28$ cells, suspended in $100 \mu \mathrm{L}$ of PBS, into the flanks of 6-week-old BALB/c nude mice (Orient Bio Inc.). Tumor growth was monitored twice a week by measuring the width and length of tumors with calipers. Tumor volume was calculated using the formula $V=\left(a^{2} \times b\right) / 2$, where $a$ and $b$ are the width and the length in $\mathrm{mm}$, respectively. Each group contained 4 or 5 animals. These experiments were performed according to institutional guidelines for the care and use of laboratory animals.

\section{Cell proliferation assay}

Cellular DNA synthesis was measured using the bromo-deoxyuridine (BrdU) assay kit (Cell Signaling Technology, USA) according to the manufacturer's instructions. Briefly, cells were plated in 96-well plates at a density of $3 \times 10^{3}$ cells/well and incubated for 6 hr, to allow attachment of cells before BrdU addition. BrdU incorporation by the cells was quantified using a plate reader at absorbance $450 \mathrm{~nm}$. The first absorbance measurement was taken at $6 \mathrm{hr}$ after cell plating, and further measurements were taken every $24 \mathrm{hr}$ after cell plating, until $96 \mathrm{hr}$.

\section{Analysis of promoter methylation}

To assay the methylation status of the $A D C Y 3$ promoter, genomic DNA was bisulfite-treated using the EZ DNA Methylation-Gold Kit (ZYMO Research, Irvine, CA, USA). Methylation-specific PCR primers were designed using EpiDesigner (Sequenom, San Diego, CA, USA; Table S2B). A methylation-specific highresolution melting assay (MS-HRM) was performed using $10 \mathrm{ng}$ of bisulfite-treated genomic DNA and ResoLight dye in a LightCycler 480 (Roche) as described in the manufacturer's protocol. Results were analyzed using Gene Scanning software (Roche). For bisulfite sequencing, the promoter region containing $39 \mathrm{CpG}$ sites was amplified from the genomic DNA of bisulfate-treated gastric cells, cloned into the TOPO-TA vector (Invitrogen), and sequenced using the 3730xl DNA analyzer (Applied Biosystems).

To investigate the effects of promoter demethylation on $A D C Y 3$ expression, KATO III cells were incubated for $96 \mathrm{hr}$ in culture medium with or without 5-Aza-2'deoxycytidine (5-Aza-dC; Sigma Aldrich) at a final concentration of $10 \mu \mathrm{M}$, and the mRNA level of $A D C Y 3$ was measured using qRT-PCR.

\section{Statistical analysis}

The statistical significance of differences between groups was determined using Student's $t$-test. $P$-values less than 0.05 were considered statistically significant. Statistical analyses were performed using the MedCalc software (version 11.4; Mariakerke, Belgium).

\section{ACKNOWLEDGEMENTS}

This study was supported by grants from the National Cancer Center Korea (NCC 0810160 and 1110270) 


\section{Conflict of Interest Statement}

No conflicts of interest were declared.

\section{REFERENCES}

1. Jemal A, Bray F, Center MM, Ferlay J, Ward E and Forman D. Global cancer statistics. CA Cancer J Clin. 2011; 61(2):69-90.

2. Cho KB, Jeon WJ and Kim JJ. Worldwide experiences of endoscopic submucosal dissection: not just Eastern acrobatics. World J Gastroenterol. 2011; 17(21):2611-2617.

3. Nakamura K, Ueyama T, Yao T, Xuan ZX, Ambe K, Adachi Y, Yakeishi Y, Matsukuma A and Enjoji M. Pathology and prognosis of gastric carcinoma. Findings in 10,000 patients who underwent primary gastrectomy. Cancer. 1992; 70(5):1030-1037.

4. Park IS, Lee YC, Kim WH, Noh SH, Lee KS and Kim H. Clinicopathologic characteristics of early gastric cancer in Korea. Yonsei Med J. 2000; 41(5):607-614.

5. Tsubono $\mathrm{Y}$ and Hisamichi S. Screening for gastric cancer in Japan. Gastric Cancer. 2000; 3(1):9-18.

6. Ebert MP and Rocken C. Molecular screening of gastric cancer by proteome analysis. Eur J Gastroenterol Hepatol. 2006; 18(8):847-853.

7. van Hal NL, Vorst $\mathrm{O}$, van Houwelingen AM, Kok EJ, Peijnenburg A, Aharoni A, van Tunen AJ and Keijer J. The application of DNA microarrays in gene expression analysis. J Biotechnol. 2000; 78(3):271-280.

8. Kim JM, Sohn HY, Yoon SY, Oh JH, Yang JO, Kim JH, Song KS, Rho SM, Yoo HS, Kim YS, Kim JG and Kim NS. Identification of gastric cancer-related genes using a cDNA microarray containing novel expressed sequence tags expressed in gastric cancer cells. Clin Cancer Res. 2005; 11(2 Pt 1):473-482.

9. Nam S, Lee J, Goh SH, Hong SH, Song N, Jang SG, Choi IJ and Lee YS. Differential gene expression pattern in early gastric cancer by an integrative systematic approach. Int $\mathrm{J}$ Oncol. 2012; 41(5):1675-1682.

10. Defer N, Best-Belpomme $M$ and Hanoune J. Tissue specificity and physiological relevance of various isoforms of adenylyl cyclase. Am J Physiol Renal Physiol. 2000; 279(3):F400-416.

11. Pierre S, Eschenhagen T, Geisslinger G and Scholich K. Capturing adenylyl cyclases as potential drug targets. Nat Rev Drug Discov. 2009; 8(4):321-335.

12. Bakalyar HA and Reed RR. Identification of a specialized adenylyl cyclase that may mediate odorant detection. Science. 1990; 250(4986):1403-1406.

13. Yang B, He B, Abdel-Halim SM, Tibell A, Brendel MD, Bretzel RG, Efendic S and Hillert J. Molecular cloning of a full-length cDNA for human type 3 adenylyl cyclase and its expression in human islets. Biochem Biophys Res
Commun. 1999; 254(3):548-551.

14. Wong ST, Trinh K, Hacker B, Chan GC, Lowe G, Gaggar A, Xia Z, Gold GH and Storm DR. Disruption of the type III adenylyl cyclase gene leads to peripheral and behavioral anosmia in transgenic mice. Neuron. 2000; 27(3):487-497.

15. Bernhard EJ, Gruber SB and Muschel RJ. Direct evidence linking expression of matrix metalloproteinase $9(92-\mathrm{kDa}$ gelatinase/collagenase) to the metastatic phenotype in transformed rat embryo cells. Proc Natl Acad Sci U S A. 1994; 91(10):4293-4297.

16. Emmert-Buck MR, Roth MJ, Zhuang Z, Campo E, Rozhin J, Sloane BF, Liotta LA and Stetler-Stevenson WG. Increased gelatinase A (MMP-2) and cathepsin B activity in invasive tumor regions of human colon cancer samples. Am J Pathol. 1994; 145(6):1285-1290.

17. Resende C, Thiel A, Machado JC and Ristimaki A. Gastric cancer: basic aspects. Helicobacter. 2011; 16 Suppl 1:38-44.

18. Shi Y, Hu Z, Wu C, Dai J, Li H, Dong J, Wang M, Miao X, Zhou Y, Lu F, Zhang H, Hu L, Jiang Y, Li Z, Chu M, Ma $\mathrm{H}$, et al. A genome-wide association study identifies new susceptibility loci for non-cardia gastric cancer at 3q13.31 and 5p13.1. Nat Genet. 2011; 43(12):1215-1218.

19. Zang ZJ, Cutcutache I, Poon SL, Zhang SL, McPherson JR, Tao J, Rajasegaran V, Heng HL, Deng N, Gan A, Lim KH, Ong CK, Huang D, Chin SY, Tan IB, Ng CC, et al. Exome sequencing of gastric adenocarcinoma identifies recurrent somatic mutations in cell adhesion and chromatin remodeling genes. Nat Genet. 2012; 44(5):570-574.

20. Kawakami H, Okamoto I, Arao T, Okamoto W, Matsumoto K, Taniguchi H, Kuwata K, Yamaguchi H, Nishio K, Nakagawa K, Yamada Y. MET amplification as a potential therapeutic target in gastric cancer. Oncotarget. 2013; 4(1):9-17.

21. Kim SJ, Hwang JA, Ro JY, Lee YS, Chun KH. Galectin-7 is epigenetically-regulated tumor suppressor in gastric cancer. Oncotarget. 2013; 4(9): 1461-1471.

22. Vecchi M, Nuciforo P, Romagnoli S, Confalonieri S, Pellegrini C, Serio G, Quarto M, Capra M, Roviaro GC, Contessini Avesani E, Corsi C, Coggi G, Di Fiore PP and Bosari S. Gene expression analysis of early and advanced gastric cancers. Oncogene. 2007; 26(29):4284-4294.

23. Yu SJ, Yu JK, Ge WT, Hu HG, Yuan Y and Zheng S. SPARCL1, Shp2, MSH2, E-cadherin, p53, ADCY-2 and MAPK are prognosis-related in colorectal cancer. World J Gastroenterol. 2011; 17(15):2028-2036.

24. Cho-Chung YS, Nesterova M, Becker KG, Srivastava R, Park YG, Lee YN, Cho YS, Kim MK, Neary C and Cheadle C. Dissecting the circuitry of protein kinase A and cAMP signaling in cancer genesis: antisense, microarray, gene overexpression, and transcription factor decoy. Ann N Y Acad Sci. 2002; 968:22-36.

25. Loffler I, Grun M, Bohmer FD and Rubio I. Role of cAMP in the promotion of colorectal cancer cell growth by prostaglandin E2. BMC Cancer. 2008; 8:380. 
26. Yamamizu K, Fujihara M, Tachibana M, Katayama S, Takahashi A, Hara E, Imai H, Shinkai Y and Yamashita JK. Protein kinase A determines timing of early differentiation through epigenetic regulation with G9a. Cell stem cell. 2012; 10(6):759-770.

27. Rosenberg D, Groussin L, Jullian E, Perlemoine K, Bertagna $\mathrm{X}$ and Bertherat J. Role of the PKA-regulated transcription factor CREB in development and tumorigenesis of endocrine tissues. Ann N Y Acad Sci. 2002; 968:65-74.

28. Sakamoto KM and Frank DA. CREB in the pathophysiology of cancer: implications for targeting transcription factors for cancer therapy. Clin Cancer Res. 2009; 15(8):2583-2587.

29. Xie S, Price JE, Luca M, Jean D, Ronai Z and Bar-Eli M. Dominant-negative CREB inhibits tumor growth and metastasis of human melanoma cells. Oncogene. 1997; 15(17):2069-2075.

30. Uren A and Toretsky JA. Pediatric malignancies provide unique cancer therapy targets. Curr Opin Pediatr. 2005; 17(1):14-19.

31. Jiang P, Enomoto A and Takahashi M. Cell biology of the movement of breast cancer cells: intracellular signalling and the actin cytoskeleton. Cancer Lett. 2009; 284(2):122-130.

32. Morimoto D, Yoshida D, Noha M, Suzuki M, Osamura RY and Teramoto A. Phosphorylation of cAMP response element binding protein (CREB) as a marker of hypoxia in pituitary adenoma. J Neurooncol. 2006; 79(2):143-150.

33. James MA, Lu Y, Liu Y, Vikis HG and You M. RGS17, an overexpressed gene in human lung and prostate cancer, induces tumor cell proliferation through the cyclic AMPPKA-CREB pathway. Cancer Res. 2009; 69(5):2108-2116.

34. Zhang D, Ma QY, Hu HT and Zhang M. beta2-adrenergic antagonists suppress pancreatic cancer cell invasion by inhibiting CREB, NFkappaB and AP-1. Cancer Biol Ther. 2010; 10(1):19-29.

35. Huhtala P, Chow LT and Tryggvason K. Structure of the human type IV collagenase gene. J Biol Chem. 1990; 265(19):11077-11082.

36. Yavartanoo M, Choi JK. ENCODE: A Sourcebook of Epigenome and Chromatin Language. Genomics Inform. 2013; 11(1):2-6

37. Sole X, Bonifaci N, Lopez-Bigas N, Berenguer A, Hernandez $\mathrm{P}$, Reina $\mathrm{O}$, Maxwell CA, Aguilar $\mathrm{H}$, Urruticoechea A, de Sanjose S, Comellas F, Capella G, Moreno V and Pujana MA. Biological convergence of cancer signatures. PLoS One. 2009; 4(2):e4544.

38. Riehl A, Bauer T, Brors B, Busch H, Mark R, Nemeth J, Gebhardt C, Bierhaus A, Nawroth P, Eils R, Konig R, Angel $\mathrm{P}$ and Hess J. Identification of the Rage-dependent gene regulatory network in a mouse model of skin inflammation. BMC Genomics. 2010; 11:537.

39. Goh SH, Hong SH, Hong SH, Lee BC, Ju MH, Jeong JS, Cho YR, Kim IH and Lee YS. eIF3m expression influences the regulation of tumorigenesis-related genes in human colon cancer. Oncogene. 2010; 30(4):398-409. 